\title{
Les effets perçus du stress chez le personnel de la santé dans le contexte actuel de la pandémie COVID-19
}

\author{
Bajji Raissoune, Lalaoui Siham \\ Laboratoire de recherche en sciences de gestion des organisations, École Nationale de Commerce et de Gestion, Kénitra, Maroc
}

\begin{abstract}
Résumé : Ce travail de recherche apporte des éléments de réponse au soulèvement qui tente d'entrevoir les retentissements de l'épisode épidémique sur le niveau de stress ressenti et sur la qualité de vie du personnel de la santé. Il s'intéresse aux aspects de l'organisation du travail du personnel de la santé et leur portée stressogène. Un important niveau de stress déjà présent que l'actuel crise sanitaire est venue rehausser. C'est la raison pour laquelle l'intérêt d'appréhender le stress perçu auprès du personnel de la santé a émergé, et c'est ce sur quoi porte cet article à travers une étude terrain menée auprès de professionnel en service au cours de la crise Covid-19 (médecins, infirmiers, dentistes, pharmaciens, techniciens de laboratoires...). Pour s'y faire, nous présenterons d'abord l'état des connaissances autour du stress professionnel, s'en suivra une présentation des caractéristiques organisationnels propres aux personnels de la santé, avant de finir avec la présentation et la discussion des résultats de l'étude menée. Des résultats qui démontrent un taux très élevé de prolifération du stress professionnel.
\end{abstract}

Mots-clés : Stress professionnel, Personnel de la santé, Performance, Corona virus.

\section{INTRODUCTION}

Au croisement des risques psychosociaux et de la sphère professionnelle se retrouve un risque professionnel largement répandu qu'est le stress professionnel. Un risque qui mobilise l'intérêt des recherches en sciences sociales en ce qu'il met au défi la santé organisationnelle et individuelle. Le stress a été l'objet de nombreuses études voulant comprendre sa complexité. Des modalisations cherchant à développer la relation entre stresseurs, à saisir les symptômes dénonciateurs de stress ou à concevoir une approche physiologique de son mécanisme.

Le stress est avant tout une réaction biologique du corps face à une situation jugée menaçante. Dans le cadre du travail, le stress est provoqué par des agents stressants propres à l'environnement professionnel. À l'ère de l'actuel crise sanitaire, une étude chinoise a démontré que plus de la moitié des 1200 participants témoigne d'un impact psychologique modéré à sévère dont d'important niveaux de stress, d'anxiété et de dépression (Qiu, et al., 2020). D'autres conclusions ont pu être relevé en affirmant que les symptômes de stress ne prendront pas forcément fin avec la diminution de la courbe de l'épidémie.

Au part delà de cela, le système de santé est l'un des principaux acteurs de résilience sociale en temps de crise (Van der Linde, 2018). Le système de santé est un regroupement de savoir-faire humain matérialisé par des techniques chirurgicales, des interventions de soins, des diagnostics et des souscriptions. Mais face à une demande en soin très importante, le personnel de santé témoigne d'une pression professionnelle excessive. Une situation qui laisse entrevoir d'importantes éventualités de prolifération du stress professionnel. 
Les motivations du stress professionnel varient et dépendent des spécificités de chaque métier. En ce qui concerne les professionnels de la santé, les sollicitations émotionnelles sont fortement dominantes. Entre accueil et prise en charge de patients malades et gravement atteints, une charge de travail importante accompagnée de pratiques répétitives, un temps de récupération très réduit, des interruptions très récurrentes, un travail à caractère urgent, divers paramètres se croisent et font que la pression perçue est excessivement stressante.

C'est donc là tout l'intérêt de cet article qui tente de soupeser le degré de propagation du stress professionnel auprès du personnel de la santé. Afin de répondre profusément aux préoccupations sociales et scientifiques de ce travail, ce papier portera en premier lieu sur un développement conceptuel autour du stress professionnel, avant de présenter les facteurs de stress spécifiques au personnel de la santé. Il sera ensuite question de présenter la méthodologie adoptée et les résultats obtenus, avant de pouvoir discuter les apports de notre étude.

\section{MiSE EN CONTEXTE}

Focaliser sur l'aspect technique et matériel du travail au détriment des considérations sociales et psychologiques impacte négativement la vie au travail. Du contexte d'évolution professionnel, technique et social découle des risques psychosociaux majeurs portant atteinte à la santé physique et mentale et au niveau de rendement. Tel est le cas du personnel de la santé qui évolue dans un cadre professionnel psychologiquement contraignant et dont le risque de prolifération d'un haut niveau de stress est prédominant. D'autant plus que dans le contexte actuel de la pandémie Covid-19, le personnel de la santé encourt davantage de risques professionnels émanant du contexte lui-même et des nouvelles conditions de travail.

C'est pourquoi cette étude s'est fixée comme objectif d'apprécier le niveau de stress chez le personnel de la santé dans le contexte actuel de la pandémie Covid-19. Elle dresse donc comme objectif de démontrer que le personnel de la santé subit un important niveau de stress professionnel susceptible de le faire osciller d'un acteur de soin à un objet de soin. Cet article tente donc de répondre à la problématique suivante : En quoi cet épisode épidémique inédit impact le niveau de stress du personnel de la santé ? D'autres questions de recherche se sont naturellement dégagées et auxquelles des éléments de réponse vont être apportés et sont :

- Quels facteurs de stress spécifiques à cette période pandémique peuvent être dégagés?

- De quelle manière les conditions actuelles du travail impactent le rendement du personnel de la santé ?

- Quels ont été les stratégies d'ajustement adoptées par le personnel de la santé ?

- À travers l'étude terrain réalisée, nous allons affirmer ou infirmer l'hypothèse que nous émettons qui stipule que le personnel de la santé connait un haut niveau de stress au cours de l'actuel crise sanitaire.

\section{LE STRESS AU TRAVAIL : REVUE DE LITTERATURE}

\section{A. Autour du stress professionnel}

Le stress professionnel est une notion qui a fait couler beaucoup d'encre et qui a suscité l'intérêt des chercheurs, des gestionnaires et de l'ensemble de la population. C'est un terme polysémique qui fait référence à la fois à la cause d'une tension, à la réaction à une contrainte et à une émotion à gérer (Loriol, 2006). Cette nature plurivoque trouve ses origines dans la genèse même du concept qui fut utilisé pour la première fois en sciences physiques par Robert Hoocke, avant qu'il soit emprunté par la médecine avec Claude Bernard et par la psychologie avec Richard Lazarus et Susan Folkman. Le stress a pu s'enrichir de la pluridisciplinarité dont il provient pour finalement désigner une pression issue d'un écart entre les exigences d'un environnement et les ressources dont dispose une personne pour y riposter (Lazarus \& Folkman, 1984). 
Le stress professionnel est donc l'un des risques psychosociaux auxquels font face les individus durant leurs prises de fonction et constitue la résultante d'une exposition à des stresseurs organisationnels (Agence européenne pour la sécurité et la santé au travail ,2014). Plusieurs approches ont tenté d'appréhender le stress au travail dont principalement l'approche biologique qui présente le stress comme étant une riposte hormonale et neurophysiologique du corps suite à une menace environnementale (Selye, 1975). Un état de stress est ici un ensemble de changements physiques et physiologiques provoqués par une situation stressante. Cette approche ne s'intéresse pas à la nature du stresseur en avançant que la réaction du corps est une réponse non spécifique, préconfigurée et dénommée «syndrome général d'adaptation ». Initialement expliqué à travers cette approche biologique, le stress faisait référence à une relation de cause à effet de sorte à ce qu'une situation stressante conduit à une réaction biologique (Selye, 1975). Cependant, la vision unidimensionnelle défendue par l'approche biologique du stress fut contestée suite à l'émergence de l'explication psychosociale du stress. Une approche qui défend la thèse d'une interaction réciproque entre l'individu et son environnement (Lazarus \& Folkman, 1984). Une interaction qui provoque un état de stress quand les ressources disponibles sont jugées insuffisantes pour faire face aux exigences environnementales.

Ainsi, face à une situation, l'individu opère une double évaluation. Il évalue d'un côté le potentiel stressant de la situation qui se présente, en plus d'évaluer les ressources dont il dispose afin de contrer la menace (Lazarus \& Folkman, 1984). Face à une situation donnée, l'individu opère une évaluation de l'enjeu qu'elle peut potentiellement détenir, et ce de manière inconsciente. Les auteurs évoquent même une double évaluation : une primaire et l'autre secondaire (Lazarus \& Folkman, 1984). L'évaluation primaire consiste à apprécier le potentiel danger d'une situation. Dépendamment de la présence d'une menace ou non, l'individu porte une seconde évaluation des possibilités de réponses dont il dispose afin d'affronter le danger. Les deux évaluations dépendent de références personnelles propres à chaque individu. C'est pourquoi le caractère stressogène d'une situation diffère d'une personne à une autre puisqu'il est influencé par le jugement porté par la personne (Scherer, 2009). Un jugement qui dépend des caractéristiques propres à l'individu où c'est l'interaction entre ses caractéristiques et celles d'une situation qui déclenche le processus de stress. Dans la même optique, il est possible de distinguer entre demandes internes (motivations personnelles, ambitions...) et ressources internes (capacités cognitives, compétences...) d'un côté, et demandes externes (exigences professionnelles, productivité...) et ressources externes (outils, assistance...) de l'autre (Mackay \& Cooper, 1987).

Selon le «effort-reward imbalance model », le stress s'opère quand une situation présente un déséquilibre entre les efforts fournis et les récompenses obtenues (Siegrist, 1996). Un effort est intrinsèque quand il s'agit d'un état d'investissement et d'engagement au travail visant à être estimé dans son environnement. L'effort peut être aussi extrinsèque et réfère aux contraintes de type professionnelles comme la charge de travail ou la fréquence des interruptions. Les récompenses quant à elles peuvent être soit des gratifications financières (augmentation salariale et primes), soit des gratifications émotionnelles (estime et reconnaissance sociale), soit des récompenses statutaires (promotion et sécurité d'emploi) (Jackson \& Schuler, 1995). L'évaluation du caractère stressogène de la situation est donc une évaluation subjective des efforts consentis par rapport aux récompenses perçues. Si les récompenses perçues sont inférieures aux efforts consentis, c'est que l'effort réalisé a été sous-évalué (Siegrist, 1996). Et c'est à partir de là que le stress professionnel prend le dessus.

Aux approches précédentes se joignent le « Job Demand-Control Model » et le « Person-Environnement Fit » qui s'intéressent à l'adéquation entre la personne et son environnement. Le Job Demand-Control Model est une approche qui étudie les conditions psychosociales présentes dans le lieu de travail à travers deux dimensions que sont la latitude décisionnelle et les exigences du travail (Karasek, 1979). Le job demand réfère aux exigences psychologiques du travail qui peuvent être issues d'une demande physique, intellectuelle ou émotionnelle. Le job control réfère au degré d'autonomie et de liberté de décision quant à l'organisation et 
l'exécution du travail. Ces deux variables permettent d'estimer la charge psychologique du travail où une faible latitude décisionnelle accompagnée de fortes exigences au travail fournissent un cadre professionnel à haut niveau de tension. Une troisième dimension fut introduite au modèle de Karasek qu'est le soutien social (Karasek \& Theorell, 1990). Ce dernier modère la relation entre les deux variables du Job Demands-Control Model que sont la latitude décisionnelle et les exigences du travail. Autrement, à travers ses interactions avec ses collègues ou ses supérieurs, l'individu perçoit un support externe dans l'accomplissement de ses missions. Un support qui permet de modérer la portée stressogène d'une importante demande environnementale et d'un faible contrôle sur le travail.

Le «Person-Environnement Fit» a été développé afin de mettre en exergue l'importance de l'interaction entre l'individu et son environnement dans l'identification du caractère stressant de cet environnement (French, et al., 1982). Selon les auteurs, il est possible de distinguer l'environnement objectif de l'environnement subjectif. L'environnement subjectif est la perception que fait une personne de son environnement objectif. L'évaluation du potentiel stressant d'une situation est de ce fait un process purement subjectif. Cette évaluation dépend de facteurs propres à la personne à savoir son histoire personnelle, sa culture, sa personnalité, son âge, ses expériences, son seuil de vulnérabilité etc. Certaines caractéristiques individuelles font en sorte d'atténuer la frustration ressentie alors que d'autres tendent à l'accentuer. Le « Person-Environnement Fit » analyse donc le fit ou le misfit de l'individu et de son environnement. Le misfit est une situation de non adéquation causée soit par des exigences excessives (adéquation positive) soit par des exigences insuffisantes (adéquation négative) (French, et al., 1982). Selon ce modèle, le stress est une tension exclusivement d'origine subjective modérée par le degré de résistance individuelle et par la nature des relations interpersonnelles.

Quant aux stresseurs organisationnels, ils font référence au contexte et aux conditions de travail. Autrement, le stress professionnel est dû soit à la façon dont le travail est conçu, soit à la façon dont l'organisation est gérée, ou les deux réunis (Cox, et al., 2000). Il s'agira alors de relations interpersonnelles détériorées, d'une communication biaisée, d'une répartition incohérente des tâches, d'une mauvaise définition des rôles, d'une faible latitude décisionnelle, de la non valorisation des efforts fournis, d'un changement organisationnel déstabilisant ou de la non prise en considération de la santé et sécurité au travail (Cox, et al., 2000). Toutes ces circonstances réunies, l'individu voit sa satisfaction professionnelle se délabrer, sa performance se dégrader, et son bien-être se détériorer (Brun \& Lamarche, 2006). Y résulte donc des préjudices sur la santé physique (troubles musculosquelettiques, problèmes cardiaques, hypertension, ulcères...) et psychique (dépression, troubles de sommeil, manque de concentration, irritabilité, burn-out...) de l'individu, et sur la santé organisationnelle de son institution (absentéisme, démissions, conflits, accidents de travail...).

Enfin, l'individu tente de s'ajuster à la pression de son environnement afin de rétablir son équilibre interne perturbé par l'intervention du stresseur. Pour se faire, l'individu a recours aux stratégies d'adaptation ou d'ajustement qui tiennent une place primordiale dans le processus de stress (Lazarus \& Folkman, 1988). C'est ce à quoi réfère le coping qui renvoie aux processus adoptés par l'individu afin de réduire la tension ressentie à la suite d'une situation de stress. L'individu a recours à des stratégies de coping soit de façon à affronter le problème pour le résoudre, soit en tentant de maîtriser les émotions induites pour pouvoir atténuer la tension émotionnelle ressentie, soit en cherchant un soutien social ou un réconfort auprès de son cercle social (Côté, 2013).

\section{B. Exploration du stress professionnel chez le personnel de la santé}

Le stress a lieu quand une personne conscientise autour de l'existence d'un danger, d'un risque ou d'une menace pour son intégrité et une réaction psychophysiologique d'adaptation se déclenche par conséquent. Le 
stress est de ce fait provoqué suite à la constatation d'une insuffisance des ressources disponibles (Cox, 1993). Par projection au monde professionnel, le stress professionnel fait désormais partie intégrante des caractéristiques de l'environnement contemporain du travail. Cependant, la particularité du stress professionnel est qu'il peut facilement osciller du normal au pathologique. Il engendre des comportements dysfonctionnels conduisant, à termes, à un épuisement professionnel.

Qu'ils soient en officines, en laboratoires, en structures hospitalières, en centres de soin ou en structures privées, les professionnels de la santé sont inondés de facteurs à haut potentiel stressogène. La spécificité des exigences professionnelles du personnel de la santé en dit long sur les contraintes psychosociales dont ils font l'objet. Entre sollicitations mentales, physiques, émotionnelles et affectives, ces professionnels constituent une population particulièrement sujette aux risques psychosociaux, notamment le stress professionnel (Laraqui, et al., 2019).

Le management du capital médical détient plusieurs spécificités propres à la nature inhérente de leur travail. L'environnement d'évolution du personnel de la santé est à la fois complexe et compliqué. Ce professionnel dispose de responsabilités dont la nature exige des charges de travail conséquentes, avec des heures de travail atypiques. Une surcharge de travail dû essentiellement à une importante demande en soin de la part de patients qui, en plus, sont devenus plus exigeants (Laraqui, et al., 2019). Un temps de travail intense en raison d'un volume de travail imposant et un temps de travail allongé en raison des nombreuses heures de travail supplémentaire à fournir constituent l'une des particularités professionnelles à fort caractère stressant.

L'insatisfaction quant aux contextes et conditions du travail vient s'ajouter à ce qui précède. Cela étant dû à une ambiance souvent tendue, des interruptions fréquentes, une urgence de prise de décision et une obligation de gérer des situations d'urgences qui marquent le quotidien du personnel de la santé (Truchot, 2004). À cela s'additionne une pénurie des ressources humaines associée à un manque de ressources matérielles. Un tout qui fait que ces professionnels perçoivent que la rémunération reçue ne couvre pas les efforts fournis. Une caractéristique stressante dans le sens où l'équilibre efforts-récompenses n'est pas respecté (Siegrist, 1996).

D'un autre côté, le personnel de la santé est en contact fréquent et quotidien avec le risque, notamment le risque infectieux. Il est aussi en contact permanent avec la souffrance aggravé, dans certain cas, par des postures d'impuissance face à certaines situations particulières de souffrance. S'y ajoute une relation soignant-soigné parfois difficile et conflictuelle où le professionnel de la santé est amené à exprimer des états émotionnels ne correspondant pas à son réel état émotionnel. Somme toute, le personnel de la santé est submergé dans un environnement qui crée une réelle prédisposition au stress professionnel.

Un travail qui nécessite une hypervigilance, un surinvestissement et une grande exigence envers soi-même. Une nature du travail qui est donc caractérisée par une forte demande psychologique accompagnée d'importants efforts à fournir, le tout sous une autonomie limitée. Telle qu'avancé par Karasek, une faible latitude décisionnelle associée à d'importantes exigences psychologiques annoncent une charge psychologique contraignante (Karasek, 1979). De plus, l'équilibre vie professionnelle-vie familiale est quant à lui extrêmement perturbé chez cette population (Le Floch, et al., 2005). Tous les critères d'un environnement hautement stressant sont donc présents.

Au par-delà de cela, et à cause de la récente crise sanitaire du corona virus, l'environnement d'évolution du personnel de la santé a connu davantage de perturbations. Une redistribution des tâches est venue s'imposer de manière urgente et rapide de tel sorte à ce que les actes non urgents ont été reportés, les circuits ont été raccourcis, les ressources humaines et matérielles ont été réorganisées, des structures ont été réaffectées... À cela s'ajoute le devoir de confronter des situations inédites dans un contexte où les recommandations se 
Bajji et al. : Les effets perçus du stress chez le personnel de la santé dans le contexte actuel de la pandémie...

succédaient et pouvaient même se contredire. Le personnel de la santé s'est retrouvé submergé dans des conditions qui ont mis à l'épreuve leur plasticité et leurs capacités d'adaptation.

Place donc aux choix méthodologiques que nous voulons justifier dans la partie qui suit.

\section{METHODOLOGIE DE RECHERCHE ADOPTEE}

Cette enquête s'insère dans le cadre d'une étude portant sur le diagnostic de l'effet du stress chez le personnel de la santé en cette période de crise sanitaire provoquée par le nouveau corona virus « Covid-19». Le but est de pouvoir apprécier la prolifération de facteurs stressants liés à leurs professions, cerner leur vécu en termes de manifestations physiques et psychologiques du stress professionnel, et évaluer leur conscience quant à la gestion du stress professionnel. Elle s'inscrit, in fine, dans une perspective d'optimisation de la gestion prévisionnelle des situations de crises. Ainsi, et afin de mener à bien les objectifs assignés à notre étude, nous avons mené une enquête terrain auprès du personnel appartenant au secteur de la santé marocain. Nous avons veillé à ce que l'échantillon soit choisi de manière à ce qu'il soit représentatif de la population étudiée. Plus de 180 personnes ont manifesté leur intérêt.

Nous avons donc interrogé 180 personnes appartenant au secteur de la santé marocain. L'étude s'est voulu d'être un examen du vécu du personnel de la santé dans leur milieu d'évolution habituel. Le recueil des données a été opéré à travers l'administration d'un questionnaire dont la validité et la fiabilité ont été préalablement vérifiées. Afin de s'aligner aux directives exigées par l'état d'urgence sanitaire et pour limiter le risque de propagation du virus dû au contact avec les répondants, l'enquête a été menée en ligne de manière exclusive.

Le questionnaire tente de mesurer l'impact de la crise sanitaire liée à la Covid-19 sur le vécu du personnel de la santé en termes de stress perçu. Autrement, il sera question de mesurer les effets du nouveau contexte de travail sur le niveau de stress ressenti par cette population. Le questionnaire comporte 24 questions portant sur différents aspects comme par exemple les difficultés personnelles et professionnelles recensées, la performance individuelle rendue, le soutien social reçu et la reconnaissance ressentie.

L'étude s'inscrit dans une logique inductive relative à une analyse phénoménologique. Cette dernière se veut d'être une approche permettant d'appréhender le vécu d'une personne tout au long d'une expérience (Lamarre, 2003). L'auteur avance qu'une stratégie de recherche phénoménologique enrichie l'objet de recherche en ce qu'elle permet de faire émerger des interprétations inédites de l'expérience étudiée (Schwandt, 1994). La nature inductive de cette recherche, qui part du particulier vers le général, permettra d'établir un cadrage de l'objet de recherche à partir des observations empiriques. C'est la raison pour laquelle l'enquête s'assied sur une approche constructiviste où la réalité est «socialement construite » à travers la perception subjective de ces acteurs, ce qui s'allie parfaitement à l'objet de notre étude.

Elle a une visée descriptive mais aussi exploratoire en essayant à la fois d'allier compréhension et analyse du phénomène. Elle tente de présenter les contours du stress professionnel, en plus d'explorer les expressions tangibles de celui-ci. L'étude se veut d'approcher ce phénomène, en l'occurrence le stress professionnel, dont l'état de connaissance dans un contexte donné n'est pas suffisant. Bien que la connaissance autour du stress professionnel soit largement abondante, peu sont les études qui ont porté sur le contexte marocain, et encore, aucune ne s'est intéressée aux professionnels de la santé. À ce stade, il apparait donc judicieux de présenter les résultats de l'étude, et c'est ce à quoi s'attardera le développement qui suit. 


\section{RESUltats}

L'étude a été menée auprès d'un échantillon de 180 personnes opérant dans le secteur de la santé au Maroc. Il s'agit plus spécifiquement de 82 médecins, 41 infirmiers, 28 pharmaciens, 5 dentistes et 24 personnes opérants dans différents secteurs de la santé (assistants médicaux, manipulateurs radio, personnel de laboratoires d'analyses et préleveurs). Ils ont à $36 \%$ entre 20 et 30 ans, à $29 \%$ entre 31 et 40 ans, à $17 \%$ entre 41 et 50 ans et à $18 \%$ plus de 50 ans.

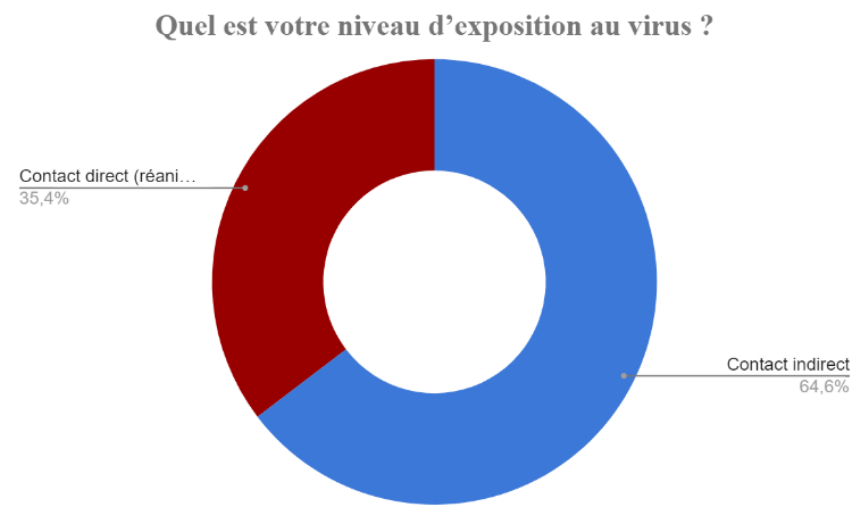

Figure 1 Répartition par genre
Comment évaluez-vous votre degré de protection lors de vos prises de fonction (sur-blouses, charlottes, bavettes, visières, cac...

Moyennement suffisant

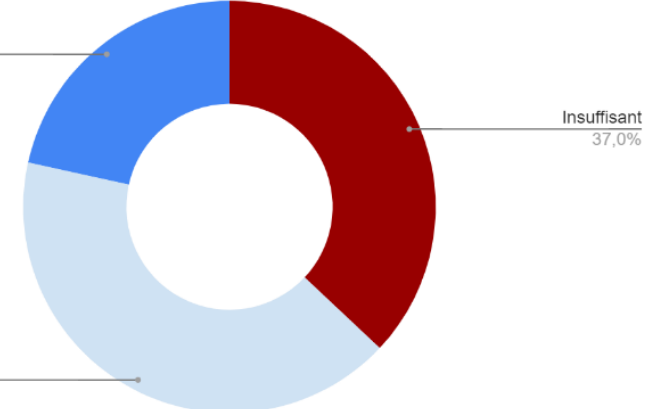

Figure 2 Répartition par métier

Il est important de préciser que 35,4\% des répondants sont en contact direct avec le virus (réanimation des patients Covid positif, urgence, dépistage...) et que 64,6\% y sont indirectement exposé. Concernant le degré de protection lors des prises de fonction (surblouses, charlottes, bavettes, visières et cache-sabot), 37\% indiquent que cette protection n'est pas suffisante voire moyennement suffisante $(41,4 \%)$, et ils ne sont que $21,5 \%$ qui affirment que la protection est bel et bien suffisante.

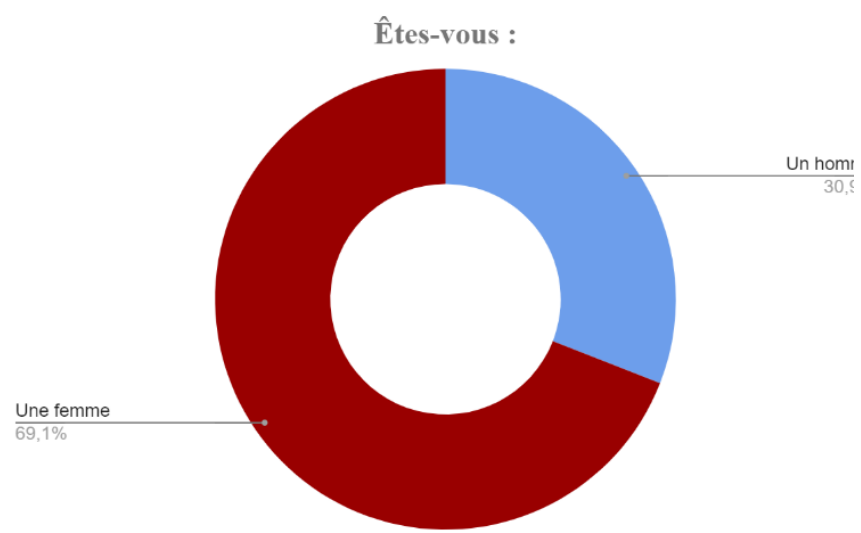

Figure 3 Niveau d'exposition au virus

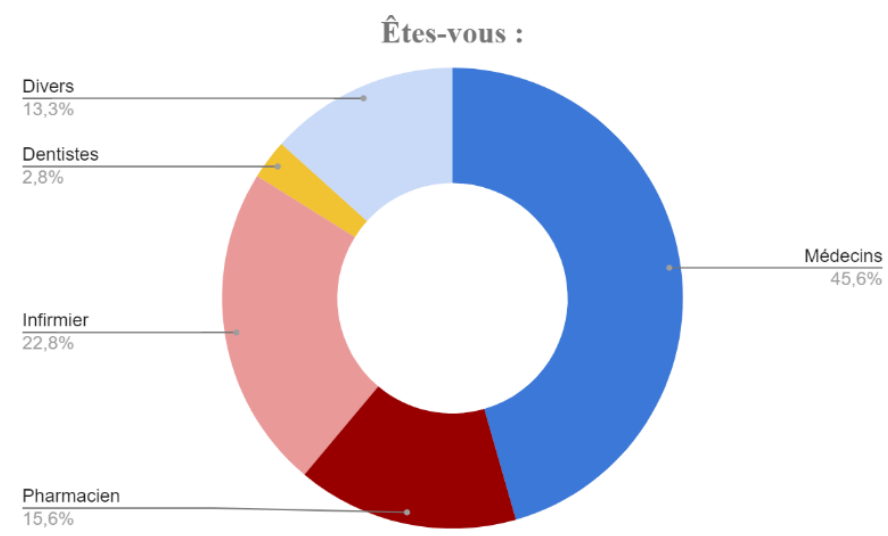

Figure 4 Degré de protection lors des prises de fonction

Durant cette crise sanitaire, le personnel de la santé encourt des risques pouvant leur porter des préjudices conséquents, ce qui constitue une source prépondérante de stress. $72,8 \%$ attestent être stressé par le risque d'être un vecteur de contamination, 56,7\% attestent être stressé par le risque d'être contaminé, 48,3\% attestent être stressé par le manque de matériel médical de protection et de soin, 47,2\% attestent être stressé par l'inquiétude quant à la gestion de plus de cas futurs de contamination, 38,9\% attestent être stressé par l'éloignement de la famille et du cercle social. Une pharmacienne avance même que « le plus dur est de répondre aux besoins d'une patientèle assez stressée par le confinement, et surtout de devoir supporter tout genre d'insultes et d'injures ». 


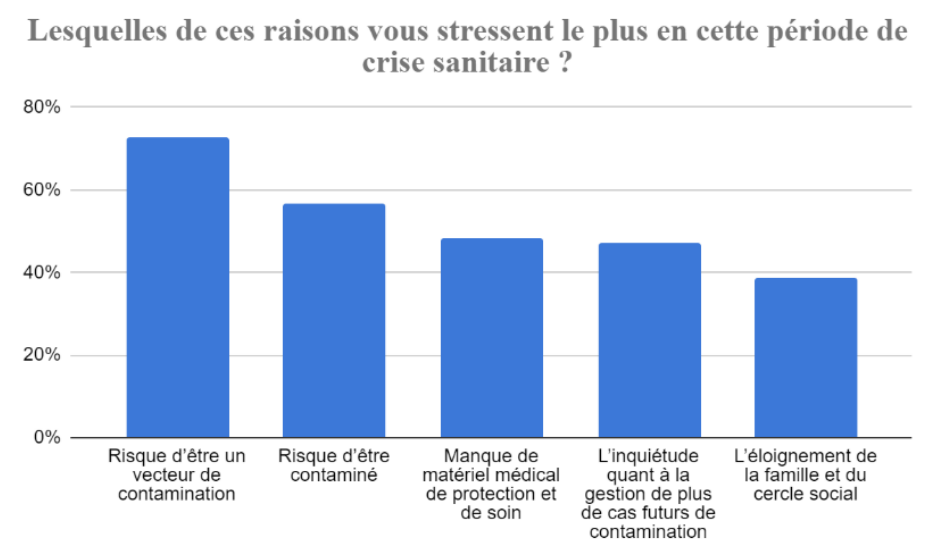

Figure 5 Les risques de stress

De surplus, ils endurent des problèmes de différentes natures, aussi bien d'ordre personnel que professionnel. Des problèmes d'isolement social reviennent à $62,2 \%$, contre $33,5 \%$ supportent des problèmes liés au déplacement, 19,5\% éprouvent des difficultés pour s'alimenter (pas de temps ni de dispositifs pour préparer leur repas durant la prise de service), et 8,5\% souffrent de difficultés pour se loger (devoir quitter la maison familiale). L'étude fait ressortir d'autres embarras comme la garde des enfants, beaucoup de réflexion et des troubles de sommeil.

À cela s'ajoute des difficultés professionnelles à savoir le manque de ressources physiques et matérielles à hauteur de 55,6\%, des difficultés de mener à bien d'autres missions médicales ne relevant pas du corona virus à hauteur de 37,2\%, des difficultés de communication avec les patients à hauteur de 33,3\% et la fait d'avoir à assurer plus de gardes que d'habitude à hauteur de 30,6\%. D'autres difficultés ont pu être relevé comme la rupture de stock de certaines fournitures de bases avec une augmentation incontrôlable des prix des produits vedette, une incompréhension des malades envers les défis qui se présentent, et même la réquisition de certains laboratoires pour y assurer des analyses Covid, comme en témoigne une biologiste interrogée.

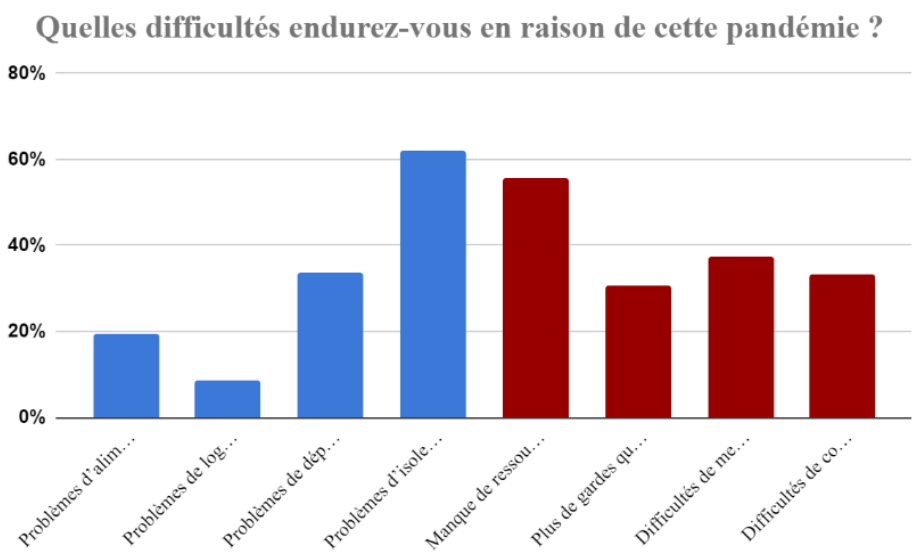

Figure 6 Les difficultés personnelles et professionnelles durant la pandémie 
Parmi les 180 personnes interrogées, 67,8\% indiquent que leur travail exige une forte mobilisation intellectuelle (attention, réflexion...). 62,2\% signalent qu'une forte mobilisation émotionnelle (ne pas s'énerver, devoir sourire, devoir réconforter...) rend leur travail épuisant. Et finalement, 49,9\% des répondants affirment devoir faire face à une forte mobilisation physique. Les interviewés ont été amené par la suite à s'exprimer par rapport à la stratégie de gestion de stress qu'ils adoptent en cette période. 47,8\% rapportent avoir recours à une stratégie de réévaluation positive qui consiste à relativiser et à dédramatiser, 29,4\% optent pour une stratégie de confrontation pour affronter le problème, $12,8 \%$ tentent de minimiser et de banaliser la situation et $10 \%$ fuient et évitent toute confrontation avec le problème afin d'assurer leur bien-être avant tout.

Qu'est-ce qui rend votre travail épuisant?

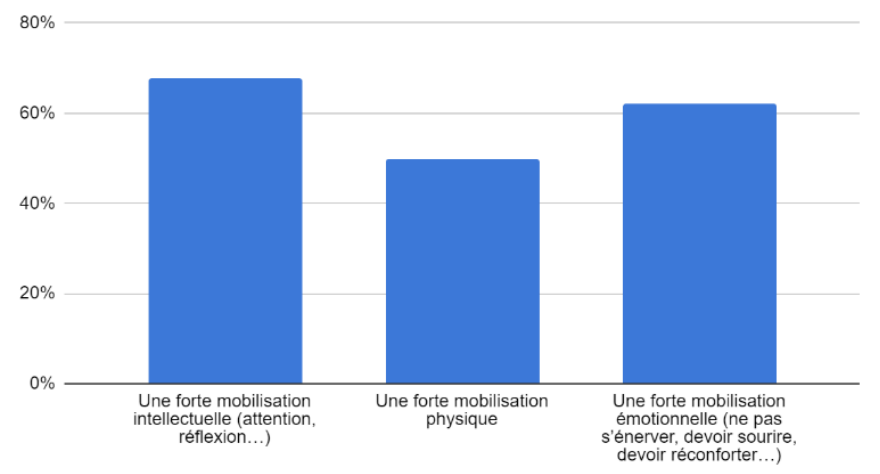

Figure 7 Épuisement au travail
Pour quelle stratégie optez-vous afin de gérer votre stress en cette période ?

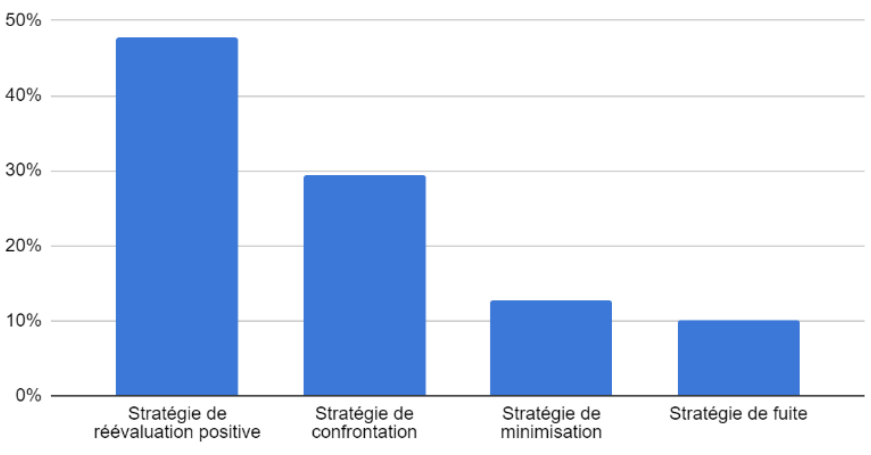

Figure 8 Stratégies de gestion de stress

Quant à l'impact des conditions actuelles de travail sur le niveau de rendement, 54,4\% affirment que cette crise a impacté négativement leur rendement et 29,4\% disent n'avoir ressenti aucun impact au niveau de leur performance rendue, alors que $16,1 \%$ attestent que la crise leur a permis d'améliorer leur rendement. Cette baisse de performance peut être expliquée par le fait que $71,1 \%$ des répondants déclarent éprouver plus de difficultés que d'habitude à réaliser leurs tâches courantes.

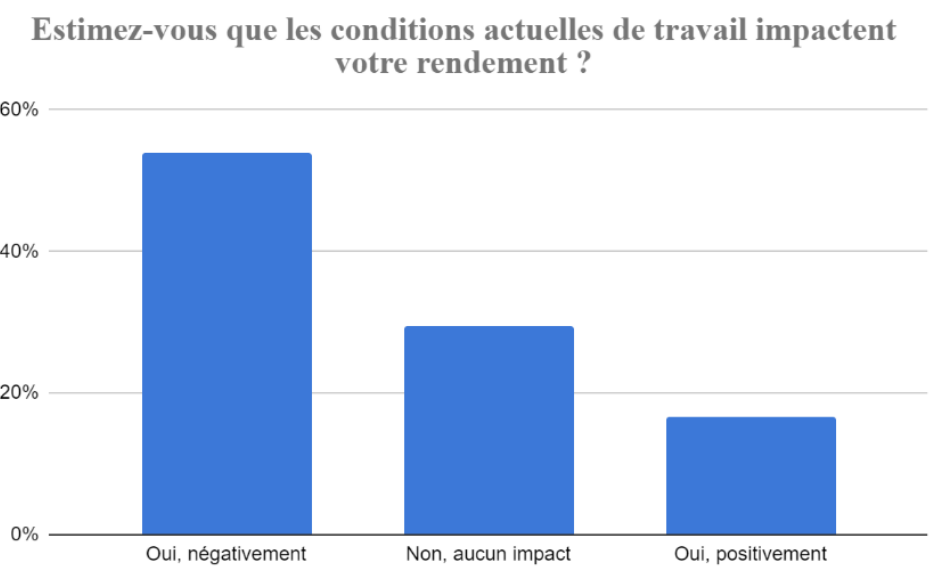

Figure 9 Impact sur le rendement

$65,2 \%$ révèlent que cette épreuve a creusé un faussé entre leur vie professionnelle et leur vie personnelle. $61,7 \%$ indiquent n'avoir aucune source de soutien psychologique, contre 38,3\% qui attestent pouvoir compter sur leurs familles, leurs amis et leur foi en dieu. Dans ce sens, 83,3\% des répondants affirment avoir recours aux valeurs spirituelles afin de s'apaiser à travers la prière $(84,3 \%)$, la lecture de versets coraniques $(53,6 \%)$ et le recours aux invocations $(26,1 \%)$. D'autres précisent qu'ils se sont armés par la méditation, le yoga, la lecture et 
même l'écriture. C'est ainsi que 50\% des répondants énoncent avoir une activité sportive régulière avant cet épisode épidémique dont $68,7 \%$ qui précisent que sa cadence a fortement baissé actuellement, 22,6\% ont vu leur cadence sportive augmentée et $8,7 \%$ ont maintenu la même cadence.

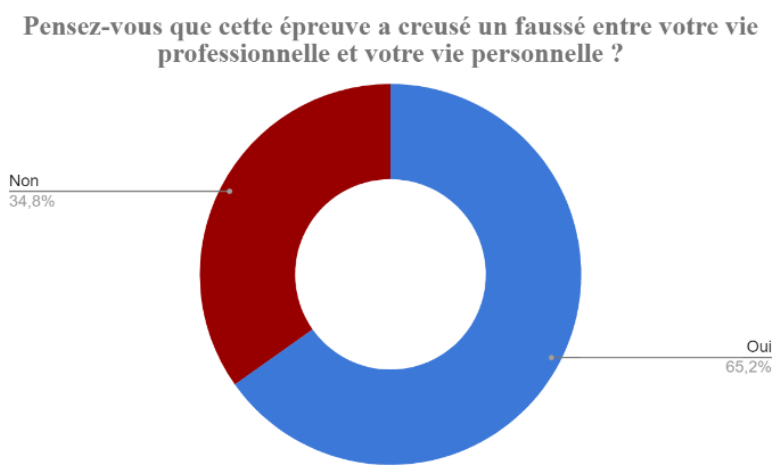

Figure 11 Équilibre vie personnelle-vie professionnelle

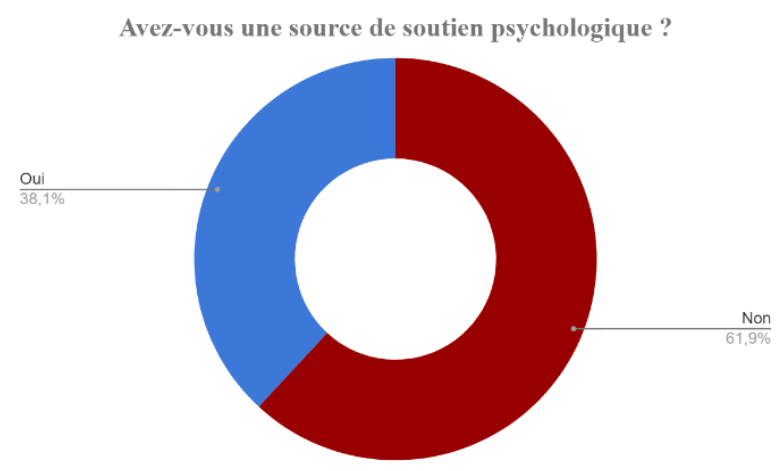

Figure 12 Soutien psychologique

D'un autre côté, $66,7 \%$ des répondants témoignent de leur véritable sens d'attachement et d'estime envers leur responsabilité en tant que garant de la santé publique en affirmant ne pas partir travailler que par obligation. Plus épatant encore, $81,2 \%$ des personnels de la santé attestent que si leur travail était un acte volontaire, ils rempliront tout de même leurs fonctions. Parmi leurs témoignages, nous pouvons citer « notre pays a vraiment besoin de nous dans ce moment critique », «c'est un devoir envers la patrie, je le fais et referai toute ma vie s'il le faut », «je me serais porté volontaire même si j'étais en retraite », « en tant qu'infirmier, j'ai choisi cette carrière pour le meilleur et pour le pire », «avec cœur, j'aime la médecine et j'aime mon travail ». De l'autre côté, les contestataires justifient leur position et admettent que «peut-être que je ne le ferais pas par obligation si j'étais célibataire et que je n'avais pas un bébé de 4 mois », « je travaille en pensant à ma famille », «j'ai prêté sermon », « on souffre d'un manque de matériel, pas de valorisation ni de la part des responsables ni de la part de la population, un rendement élevé et une rémunération très basse, en plus d'horaires de travail très atypiques », «J'aurais préféré être confinée moi aussi chez moi près de ma petite famille », « c'est un travail très stressant ».

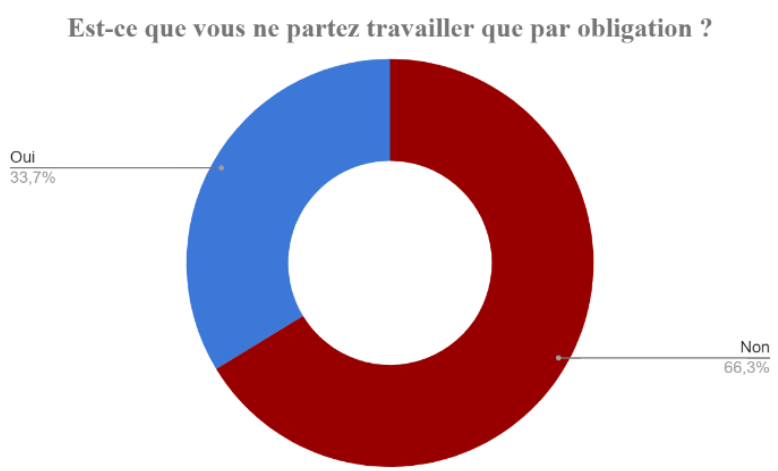

Figure 12 Travail par obligation

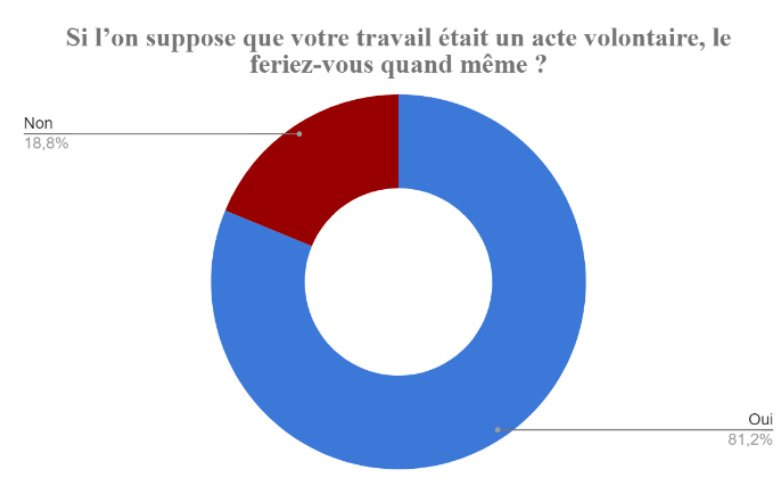

Figure 13 Travail volontaire

$56,7 \%$ du personnel de la santé assure ressentir que leurs efforts sont remarqués, appréciés et félicités par la communauté non médicale. Un médecin nous témoigne que «ce travail doit être apprécié. Les médecins risquent leur santé et celle de leur famille, travaillent plus d'heures que d'habitude, et j'en passe. C'est très difficile physiquement et psychologiquement. Nous devons obtenir le soutien de tous [...] En contrepartie, nous 
devons faire notre travail et aider». Finalement, le personnel de la santé signale qu'ils se sentent stressés, débordés, anxieux, inquiets, angoissés, émotionnellement épuisés, qu'ils manquent de repos, de sommeil, de concentration, d'attention, et qu'ils craignent énormément le futur.

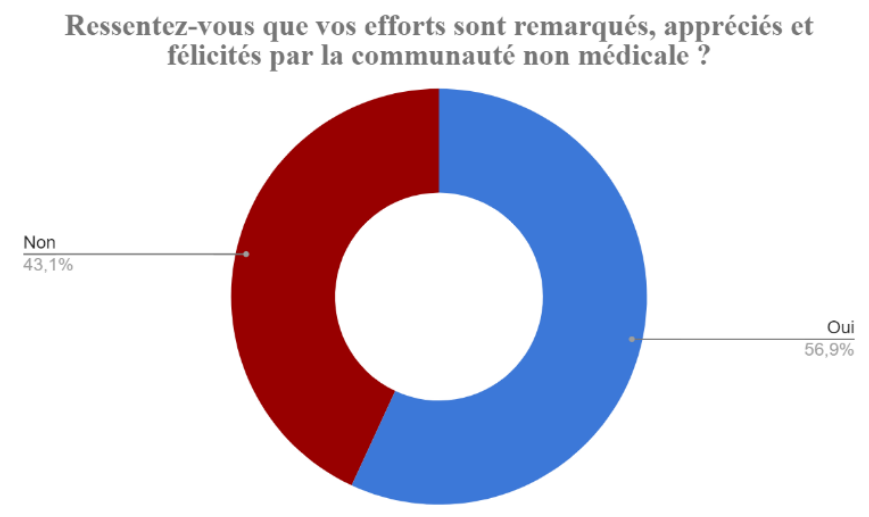

Figure 13 Appréciation des efforts par la communauté

\section{DISCUSSION}

Cette étude a permis de faire le point sur plusieurs aspects relevant du stress perçu chez le personnel de la santé dans le contexte actuel de la pandémie Covid-19. Les résultats révèlent un état de stress assez développé chez la population étudiée. De prime abord, ces agents révèlent que la nature de leur travail requiert une forte mobilisation intellectuelle (attention, réflexion...), émotionnelle (ne pas s'énerver, devoir sourire, devoir réconforter, le contact avec la mort...) et physique. Ce qui signifie que leurs capacités, qu'elles soient cognitives ou organiques, sont sollicitées, ce qui en résulte un épuisement généralisé. Cet épuisement justifie les propos de $71 \%$ des répondants qui indiquent avoir plus de mal que d'habitude à accomplir des tâches courantes et coutumières. En conséquence, le rendement des professionnels de la santé est compromis où ils sont plus que la moitié à avoir relevé une baisse de leur rendement en raison de la crise sanitaire. Cet impact négatif sur le niveau de rendement est expliqué par le fait que le stress professionnel est évalué comme étant un obstacle et une menace pour la grande majorité des répondants.

Les professionnels de la santé signalent un manque alarmant de matériel de travail et de fournitures médicales, ce qui crée des complications quant à leurs gestion. De plus, ils annoncent se sentir peu ou pas assez protégés en raison d'une pénurie d'équipements de protection (surblouses, charlottes, bavettes, visières et cachesabot). En plus d'être en contact avec le virus, cette déficience rend ces individus encore plus exposés au risque de contamination qu'ils ne le sont déjà. Le personnel de santé n'a pas manqué d'exprimer sa frustration par rapport à ce point. Plus de la moitié des répondants se sentent stressés par rapport au manque de matériel médical de protection et de soin. Ces derniers ne sont disponibles qu'à travers un stock minimum ne laissant aucune marge au personnel en cas de besoin supplémentaire. Cette rareté des matières premières crée auprès du personnel de la santé une inquiétude quant à la gestion de plus de cas futurs de contamination. De cette situation découle une prédisposition conséquente au stress professionnel. Une affirmation qui est d'autant plus confirmée par les participants de l'enquête qui affirment que le risque d'être contaminé est un facteur de stress prédominant. Il en émane le stress lié à la crainte d'être, en conséquent, un vecteur de contamination. Cette crainte a contraint le personnel de la santé à devoir s'éloigner de leur cercle social, question de les préserver. Ces conditions ont provoqué un isolement et un éloignement de la famille chez les deux tiers des répondants.

Aux côtés des contraintes précitées, d'autres difficultés viennent entachées le vécu du personnel de la santé durant cette crise sanitaire. Ces contraintes touchent aussi bien leur vie professionnelle que leur vie personnelle 
qui sera inévitablement influencée. Au-delà du manque de ressources matérielles, les enquêtés sont unanimes quant à l'insuffisance alarmante des ressources humaines dont la pandémie a enfoncé le fossé. Cette problématique fait que la charge de travail à gérer dépasse le capital humain disponible, ce qui engendre une pression et une fatigue professionnelle démesurée. Pour les médecins et infirmiers en particulier, cette problématique en crée aussi une autre relative à la gestion des autres missions médicales ne relevant pas du corona virus, vu qu'une grande partie des ressources matérielles et humaines ont été affectées aux services « Covid ». Les services classiques des structures de santé se sont, par conséquent, restreint à un effectif, à du matériel et à des disponibilités de réception très limités. Il en découle une autre complication révéler par le personnel de la santé où ces conditions ont contraint ces derniers à devoir assurer des journées de travail plus conséquentes et des gardes plus fréquentes. Toutes ces péripéties ont fait que la charge mentale et émotionnelle de ces acteurs de la santé soit extrêmement pesante physiquement mais aussi psychologiquement. D'autres éléments ont pu être recueilli comme l'incompréhension des malades envers la nature austère du défi qui se présente, ce qui crée des difficultés de communication avec les patients. Cette « irresponsabilité » complique la mission du personnel de la santé et provoque un environnement prospère à la prolifération du nombre de contamination.

Le personnel de la santé communique d'autres soucis d'ordre personnel directement engendrés par les circonstances atypiques liées à la propagation du corona virus. Comme développé ci-dessus, et voulant préserver leur entourage du risque d'être contaminé, le personnel de la santé s'est vu obligé de quitter le foyer familial vers des logements mis à leur disposition par des bénévoles. Beaucoup d'entre eux témoignent avoir fait l'objet d'une discrimination dans le sens où ils n'étaient plus les bienvenus dans leurs propres immeubles par exemple, vu que tout le voisinage craignait leur présence. S'y rajoute des difficultés de déplacement avec le recul de la cadence des transports en commun et des difficultés pour s'alimenter avec la fermeture des établissements de restauration. Sans oublier la manifestation d'embarras comportementaux et intellectuelles comme les troubles de sommeil et les surmenages, et l'installation d'un réel déséquilibre du rapport responsabilité professionnelle - responsabilité personnelle (gestion du ménage, garde des enfants, vie de couple...). C'est ainsi que $65 \%$ du personnel interrogé affirment que cette épreuve a creusé un faussé entre leur vie professionnelle et leur vie personnelle.

$\mathrm{Ne}$ disposant pas de marge de répit, les professionnels de la santé accumulent les agents stressogènes en dépit de leur bien-être et de leur épanouissement. L'étude a pu démontrée que cette catégorie socioprofessionnelle témoigne d'un important niveau de stress professionnel et d'épuisement. Un état psychologique altéré, conduisant à des troubles comportementaux et émotionnels. Pour y faire face, le personnel de la santé adoptent inconsciemment des stratégies de gestion de stress dont la réévaluation positive, la stratégie de fuite, la stratégie de minimisation et la stratégie de confrontation.

Presque la moitié d'entre eux affirment avoir recours à la réévaluation positive, un tiers rapportent avoir tendance à confronter le problème, le reste des répondants révèlent opter pour une stratégie de minimisation et de fuite. Survivre une période à très haut potentiel stressant estime la prédisposition d'un soutien psychologique, intellectuel ou technique de la part du cercle social (Karasek \& Theorell, 1990). Cependant, dans notre étude, ils ne sont qu'un quart à avoir témoigné la disponibilité d'une source de soutien social. Les enquêtés ont affirmé avoir recours à divers moyens afin de s'apaiser et retrouver leur homéostasie émotionnelle déstabilisée. Parmi ces moyens, il est possible de retrouver la méditation, le yoga, la lecture, l'écriture et l'assise spirituelle (Grout, 1980). En ce qui concerne l'activité physique comme outils de décompression et d'émancipation, les avis sont mitigés. La grande majorité des répondants parmi ceux ayant déclarée entretenir une activité sportive régulière, 
énonce qu'ils ont dû revoir à la baisse sa fréquence. Ils expliquent que c'est principalement à cause d'une disponibilité très limitée de temps à usage personnel, ou en raison d'une fatigue physique très éreintante.

Motivation et reconnaissance sont des piliers de performance. L'étude démontre que presque la moitié du personnel de la santé ne remarque pas que leurs efforts sont remarqués, appréciés et félicités par la communauté non médicale. Toutefois, la sur-sollicitation des professionnels de la santé ne les a pas empêcher d'exprimer leur incontestable sens d'attachement et d'estime envers leur métiers respectifs. La plupart d'entre eux assure fermement leur réel dévouement à la nature de leur travail qui englobe un aspect humain et social très admiré par le personnel de la santé. Cette ferveur fait que $80 \%$ des témoignages récoltés partent dans le sens où aucune marge d'hésitation n'aura lieu si du bénévolat ou du volontarisme leur soit proposé. Cette affirmation témoigne de l'implication indubitable et inconditionnelle des professionnels de la santé. D'autres positions ont été relevé parmi ceux qui déclarent ne partir travailler que par obligation et qui ne sont pas prêt à fournir d'actes volontaires. L'analyse de leurs déclarations fait ressortir des justifications où le caractère familial est dominant et où le manque de motivation désintéresse les contestataires.

Au final, et tout au long de cette crise sanitaire, le personnel de la santé fut submergé de facteurs à haut potentiel stressant. Un sentiment de perte de contrôle s'est installé, accentué par la perte de repères personnels et professionnels (Oulehri \& Rolling, 2020). Un contexte qui a nécessité une importante mobilisation quotidienne des capacités d'adaptation. Une sur-sollicitation de ces mécanismes d'ajustement qui a fait en sorte que la fatigue psychologique s'installe. Une fatigue qui devrait inéluctablement être considérée, prise en charge, et soignée.

\section{CONCLUSION}

Nous l'avons tous remarqué, le personnel de la santé a été en devanture tout au long de la crise sanitaire liée à la « Covid-19». Ils se surpassaient pour combattre le virus, se dotaient de beaucoup de courage et partaient l'affronter sans aucune réticence. Leur mobilisation générale et inédite a marqué les esprits et une vénération leur est due. Une mobilisation importante qui leur a valu la considération de tous. Ce devoir professionnel a fait que le personnel de la santé vivait une pression inégalée et une charge de travail incommensurable. Et comme si ces paramètres ne suffisaient pas, ces personnes allaient même jusqu'à parier leur vie et leur santé contre celle des citoyens. Une prise de risque sérieuse et un épuisement contraignant ont permis à des manifestations de stress professionnel de s'infiltrer. Ce dernier a rendu le personnel de la santé vulnérable face à des contraintes psychosociales, organisationnelles et physiques.

Cette crise sanitaire a permis de lever le voile sur le vécu du personnel de la santé. Leurs sacrifices, autrefois méconnus du grand public, est aujourd'hui mis en lumière. Côtoyer la mort, approcher la maladie, travailler de nuit et pleines d'autres particularités caractérisent le travail des professionnels de la santé (Duchateau, et al., 2002). La conjugaison de ces paramètres expose ces derniers à un risque psychologique contraignant qu'est le stress professionnel.

À travers cette étude, il a été démontré que le stress professionnel est fortement répandu auprès du personnel de la santé. Ce personnel est exposé de manière continue et répétée à un haut niveau de stress professionnel. Sous ces conditions de travail stressantes, cet acteur de soin vacille à un objet de soin nécessiteux d'une prise en charge imminente. Un haut niveau de stress que la crise sanitaire a davantage appuyé, comme l'a pu prouver cette étude. Un stress qui impacte le rendement et qui, à son tour, affecte le bon fonctionnement de la structure médicale. L'étude a également dégagé que le recours à des stratégies de réévaluation positive est fortement dominant et que peu d'entre eux tentent de fuir ou de minimiser la menace environnementale.

Au-delà de la recherche d'efficience, les risques psychosociaux auxquels font face le personnel de la santé est tout aussi un paramètre à considérer. Au moment où nul ne peut assurer pouvoir désinfecter le milieu 
Bajji et al. : Les effets perçus du stress chez le personnel de la santé dans le contexte actuel de la pandémie...

hospitalier des dépôts à risque de stress qu'il contient, il convient d'identifier les axes d'actions prioritaires afin d'améliorer l'environnement d'évolution du personnel de la santé. Il peut s'agir notamment de l'amélioration des conditions de travail, d'une meilleure reconnaissance et une révision des rémunérations, d'un programme de formation continue et d'amélioration des savoir-faire, d'un soutien psychologique et bien d'autres. Valoriser le capital humain au sein du système est un impératif à garantir afin de s'assurer d'un niveau de rendement optimal. Il est donc fondamental de conscientiser autour des retentissements du stress professionnel à travers des interventions ergonomiques, des mesures organisationnelles et des solutions orientées vers les individus.

La principale limite de ce travail de recherche est l'échantillon sur lequel a porté l'étude. Ce dernier, malgré qu'il ait atteint 180 répondants, reste encore faible. Élargir encore plus l'échantillon de répondant peut être une perspective à adopter afin d'assurer une meilleure sensibilité des résultats.

\section{BIBLIOGRAPHIE}

[1] Agence européenne pour la sécurité et la santé au travail . (2014). Calcul des coûts du stress et des risques psychosociaux liés au travail. Analyse documentaire, Luxembourg.

[2] Brun, J., \& Lamarche, C. (2006). Assessing the costs of work stress. A research report. Quebec: The Chair in Management of the Health and of the Security at Work in Organizations.

[3] Côté, L. (2013). Améliorer ses stratégies de coping pour affronter le stress au travail. Psychologie Québec, 30(5), 41-44.

[4] Cox, T. (1993). Stress Research and stress management: Putting theory to work. Sudbury: HSE Books.

[5] Cox, T., Griffiths, A., \& Rial-Gonzalez, E. (2000). Research on Work Related Stress. European Agency for Safety and Health at Work. Luxembourg: Office for Official Publications of the European Communities.

[6] Duchateau, F. X., Bajolet-Laplante, M. F., Chollet, C., Ricard-Hibon, A., \& Marty, J. (2002). Exposition à la violence en SMUR. Annales françaises d'anesthésie et de réanimation, 21(10), 775-778.

[7] French, J., Caplan, R., \& Harrison, R. (1982). The mechanisms of job stress and strain. New York: John Wiley $\&$ Sons.

[8] Grout, J. (1980). Stress reduction training modules for nurses. Dans K. Claus, \& J. Bailey (Éds.), Living with stress and promoting well-being: A handbook for nurses (pp. 137-199). St Louis: Mosby.

[9] Jackson, S. E., \& Schuler, R. S. (1995). Understanding Human Resource Management in the Context of Organizations and their Environments. Annual Review of Psychology, 46(1), 237-264.

[10] Karasek, R. (1979). Job demands, job decision latitude and mental strain: Implications for job design . Administrative Science Quarterly.

[11] Karasek, R., \& Theorell, T. (1990). Healthy work. Stress, productivity, and the reconstruction of working life. Basic Books.

[12] Lamarre, A. M. (2003). Étude de l'expérience de la première année d'enseignement au primaire dans une perspective phénoménologico-herméneutique. Montréal: Université du Québec.

[13] Laraqui, O., Manar, N., Laraqui, S., Hammouda, R., Deschamps, F., \& Laraqui, C. (2019). Risques psychosociaux et syndrome d'épuisement professionnel des professionnels de soins hospitaliers. Archives des Maladies Professionnelles et de l'Environnement, 80(5), 386-397.

[14] Lazarus, R., \& Folkman, S. (1984). Stress appraisal and coping. New York: Springer Publishing Company.

[15] Lazarus, R., \& Folkman, S. (1984). Stress appraisal and coping. Springer Publishing Company.

[16] Lazarus, R., \& Folkman, S. (1988). Manual for the ways of coping questionnaire. New York: Consulting Psychologists Press.

[17] Le Floch, N., Clarisse, R., Testu, F., \& Kindelberger, C. (2005). La conciliation des rôles professionnels et parentaux, un facteur de stress professionnel : Construction et première validation d'une échelle de mesure. Revue européenne de psychologie appliquée, 55, 9-20.

[18] Loriol, M. (2006). Je stresse donc je suis. Comment bien dire son mal être. Paris: Mots et Cie.

[19] Mackay, C., \& Cooper, C. (1987). Occupational stress and health : some current issues. Dans C. C. Robertson (Éd.), International Review of Industrial and Organizational Psychology (p. Volume 11). Chichester: John Wiley \& Sons Ltd. 
[20] Oulehri, W., \& Rolling, J. (2020). Covid-19, retour d'expérience de la prise en charge réanimatoire des patients et du soutien médico-psychologique aux soignants à Strasbourg, France. Revue de neuropsychologie, 12(2), 115-121.

[21] Qiu, J., Shen, B., \& al., M. Z. (2020). A nationwide survey of psychological distress among Chinese people in the Covid-19 epidemic: implications and policy recommendations. General Psychiatry.

[22] Scherer, K. (2009). Emotions are emergent processes: They require a dynamic computational architecture. Philosophical Transactions of The Royal Society B Biological Sciences, 364(1535), 3459-3474.

[23] Schwandt, T. (1994). Constructivist, Interpretivist Approaches to Human Inquiry. Dans N. Denzin, \& Y. Lincoln (Éds.), Handbook of Qualitative Research (pp. 118-137). London: Sage.

[24] Selye, H. (1975). Le stress de la vie : Le problème de l'adaptation. Editions GALLIMARD.

[25] Siegrist, J. (1996). Adverse health effects of high effort-low reward condition. Journal of Occupational Health Psychology, 1, 27-41.

[26] Truchot, D. (2004). Épuisement professionnel et burnout : concepts, modèles, interventions. Paris: Editions Dunot.

[27] Van der Linde, C. (2018). La gestion des situations sanitaires exceptionnelles et de crise. Dans P. d. l'EHESP (Éd.), Le management en santé. Gestion et conduite des organisations de santé (pp. 493-524). Rennes. 